\title{
An Efficient Virtual Topology Design and Traffic Engineering Scheme for IP/WDM Networks
}

\author{
Namik Sengezer and Ezhan Karasan \\ Department of Electrical and Electronics Engineering \\ Bilkent University, Ankara 06800, TURKEY, \\ namik, ezhan@ee.bilkent.edu.tr
}

\begin{abstract}
We propose an online traffic engineering (TE) scheme for efficient routing of bandwidth guaranteed connections on a Multiprotocol label switching (MPLS)/wavelength division multiplexing (WDM) network with a traffic pattern varying with the time of day. We first consider the problem of designing the WDM virtual topology utilizing multi-hour statistical traffic pattern. After presenting an effective solution to this offline problem, we introduce a Dynamic tRaffic Engineering AlgorithM (DREAM) that makes use of the bandwidth update and rerouting of the label switched paths (LSPs). The performance of DREAM is compared with commonly used online TE schemes and it is shown to be superior in terms of blocked traffic ratio.
\end{abstract}

Keywords:Traffic Engineering, Virtual Topology Design, MPLS, LSP, Dynamic Routing.

\section{Introduction}

MPLS architecture provides powerful features for traffic engineering [1]. The encapsulated Internet Protocol (IP) packets can be routed explicitly along virtual connections called LSPs. The explicit routing capability in MPLS combined with enhanced link state interior gateway protocols (IGPs) and resource reservation protocol (RSVP), enables the routing of the traffic flows taking into account both the quality of service and bandwidth requirement of the traffic flows and current network state such as traffic load and available capacity on the links. The extensions to RSVP allows the dynamic updates of the LSP bandwidth and construction of the new explicit route before tearing down the old route, which is useful for LSP reroutings $[2,3]$.

The rerouting of virtual connections is frequently referred in the literature as a means of TE. When it is performed on the underlying WDM layer [4], the virtual topology seen by the upper layer changes with the TE actions. When it is carried out solely on the MPLS layer, the virtual topology is kept intact and the LSPs are rerouted on the existing lightpaths $[5,6]$.

In this work, we develop a new MPLS layer TE scheme, DREAM, for dynamic connections and show that with a properly designed virtual topology, it can enhance the network performance to a large extent compared to other TE 
schemes, without changing the virtual topology. We model the dynamic traffic demand using a Multi-Hour traffic pattern which will be discussed in Section 2.1. In Section 2.2, the Multi-Hour Virtual Topology Design (MVTD) problem is investigated. A heuristic algorithm utilizing tabu search (TS) is proposed to solve this problem and the solutions are compared with upper bounds obtained by using mixed integer linear programming (MILP). DREAM is explained in Section 3, and its performance is compared with other commonly used TE schemes. Section 4 includes an extension of DREAM where multiple parallel LSP tunnels can be provisioned between any node pair.

\section{Multi-Hour Virtual Topology Design}

Multi-hour virtual topology design problem can be identified as designing the optimum MPLS layer virtual topology for a daily traffic pattern that changes over time [7]. We assume that, some basic information on the hourly traffic statistics is available. That statistical information is the estimate of the maximum traffic rate in every hour, between each node pair. In this work, it is assumed that there exists a non-zero traffic demand between any two nodes in the network for all hourly periods.

\subsection{Traffic Model}

Since flow-level traffic models are better-suited for TE purposes than packet-level models, flow level traffic statistics is utilized in this work. To model the traffic, an approach that is proposed in [8] is used, which is suitable for generating the traffic when the nodes are spread over a large geographical area. In this approach, for each node $i$, a time zone offset $\tau_{i}$ and a traffic generation ratio tgen $_{i}$ are defined. tgen $_{i}$ represents the maximum instantaneous traffic generated by node $i$. An activity function $\operatorname{act}(i, t)$ depending on these values is defined as

$$
\operatorname{act}(i, t)= \begin{cases}0.2 & \text { if } \mathrm{t}_{\text {local }}(i, t) \in[0: 00 ; 6: 00) \\ 1-0.8\left(\cos \left(\frac{\left(t_{\text {local }}(i, t)-6\right) \pi}{18}\right)\right)^{10} & \text { if } \mathrm{t}_{\text {local }}(i, t) \in[6: 00 ; 24: 00)\end{cases}
$$

In this formula, $t$ is the coordinated universal time in hours and the function $t_{\text {local }}(i, t)$ is defined as, $t_{\text {local }}(i, t)=\left(t+\tau_{i}+24\right) \bmod 24$. The activity function is illustrated in Fig. 1 for node $i$ which belongs to the universal time zone.

For a node pair $(i, j)$, the expected instantaneous traffic between these nodes in time $t$ is calculated as

$$
T_{\text {expected }}(i, j, t)=\operatorname{tgen}_{i} \times \operatorname{act}(i, t) \times \operatorname{tgen}_{j} \times \operatorname{act}(j, t)
$$

The actual instantaneous traffic between nodes $i$ and $j$ is obtained by adding a zero mean Gaussian random variable to this expected value. The standard deviation of the Gaussian random variable is given by $k$ times the expected traffic, i.e., 


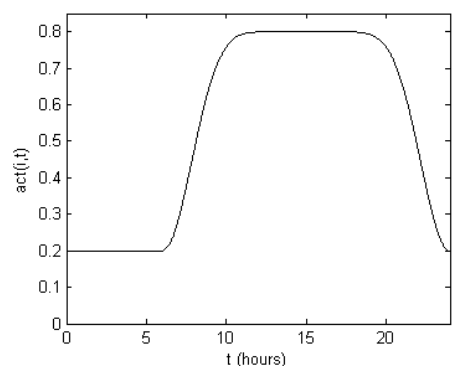

Fig. 1. Activity function

$$
T_{\text {actual }}(i, j, t)=T_{\text {expected }}(i, j, t)+N\left(0, k \times T_{\text {expected }}(i, j, t)\right)
$$

The parameter $k$ is a measure of the deviation of the actual traffic from its estimate and will be used as a parameter for evaluating the robustness of the TE schemes with respect to uncertainty in traffic estimations. The traffic generation ratio $\operatorname{tgen}_{i}$ is assumed to be uniform for all the nodes in the network and will be referred as tgen throughout the paper. In the proceeding sections, the lightpath capacities will be expressed as the ratio of their bandwidth to $\operatorname{tgen}^{2}$.

\subsection{Tabu-Search Based Multi-Hour Virtual Topology Design(TS-MVTD)}

The objective in designing the topology is to maximize the total amount of routed traffic during the day while satisfying the optical layer interface constraints. We assume that the total number of lightpaths in the virtual topology is given, and constructing the virtual topology corresponds to deciding between which source destination pairs to place these lightpaths subject to the nodal interface constraints. The nodal interface constraints correspond to the maximum number of lightpaths emanating at a node.

We develop a heuristic algorithm based on the Tabu Search procedure to solve this problem. Tabu Search is an iterative search procedure utilizing adaptive memory [9]. Its distinguishing feature from other search procedures is that nonimproving moves are also allowed to escape local optima. Entrapment in cycles is avoided by declaring the visited solutions tabu for a number of iterations and forbidding the moves leading to a tabu solution.

The statistical traffic information that is input to the algorithm consists of $H n \times n$ matrices where $H$ is number of hours in the considered interval and $n$ is the number of nodes. The traffic information for hour $h$ is represented by matrix $T^{h}$ and the entry $T_{i, j}^{h}$ in this matrix is the maximum value of the expected traffic between nodes $i$ and $j$ during the $h^{\text {th }}$ hour.

The objective function is the total amount of traffic that can be routed on the current topology in every hour. To calculate the objective function, an offline 
routing algorithm based on Dijkstra's shortest path approach is utilized. For each single hour $h$, the entries in $T^{h}$ are sorted according to their bandwidth requirement in a descending order and they are served in that order. The dynamic link weights used for computing the shortest paths are inversely proportional to the residual capacities on the links. The offline routing algorithm tries to route the traffic flows starting from the largest flow using paths with the maximum residual capacity. If more than one move give the highest objective value, a tie breaker parameter is used to choose the best move. The tie breaker parameter is calculated as $\sum_{i, j \in V} \sum_{h=1}^{H} s_{i j} T_{i, j}^{h}$ where $\mathrm{V}$ is the set of nodes and $s_{i j}$ denotes the number of hops on the shortest path between $i$ and $j$ in the resulting topology. Between moves giving the same objective value, the one with the smaller tie breaker value is chosen.

The flowchart of the TS-MVTD algorithm is given in Fig. 2. A move is defined as tearing down an existing lightpath and setting up a new lightpath between two nodes which do not share a common lightpath. A valid move is defined as a move that is not in the tabu list and results in a topology that satisfies the interface constraints. The algorithm stops if there is no improvement in the objective value for $I$ iterations.

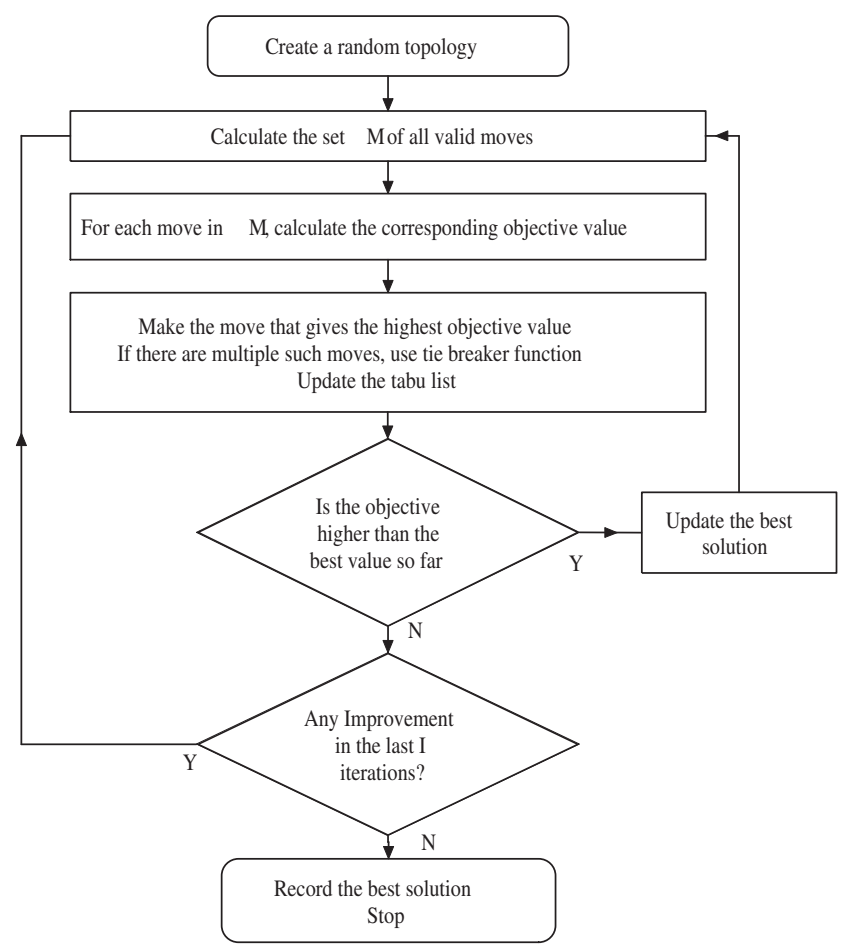

Fig. 2. Flowchart of the TS-VTDM algorithm 
To evaluate the performance of the proposed algorithm, an upper bound on the percentage of routed traffic for a given number of lightpaths that can be achieved by any algorithm is obtained by formulating the problem as an MILP problem and relaxing the integrality constraints on the flow variables. The percentages of traffic carried by each algorithm are given in Table 2.2 for different number of lightpaths. These results are obtained using a lightpath capacity $C=$ $3 \operatorname{tgen}^{2}$. According to the lower bounds, at least 15 lightpaths are needed to route all the traffic demands. Proposed TS-MVTD algorithm achieves this using 17 lightpaths, however it can route close to $100 \%$ of the traffic demands for 15 and 16 lightpaths.

Table 1. The percentages of routed traffic by the TS-MVTD algorithm and the upper bound for different number of lightpaths

\begin{tabular}{lccccc}
\hline \# of lightpaths & 13 & 14 & 15 & 16 & 17 \\
\hline upper bound & 93.403 & 97.886 & 100 & 100 & 100 \\
TS-MVTD result & 86.897 & 93.885 & 98.816 & 99.971 & 100 \\
optimality gap(\%) & 6.966 & 4.087 & 1.184 & 0.029 & 0 \\
\hline
\end{tabular}

\section{Online Traffic Engineering with Single LSP Between Two Nodes}

\subsection{Modeling Traffic Flows}

As stated in Section 2.1, a flow model is used to represent the traffic in our work. In this section, a single LSP is constructed between each source-destination pair and the changes in the traffic amount between these nodes is represented by changing the bandwidth requirement of the constructed LSP. The changes in the bandwidth requirements of the LSPs are modelled by Bandwidth update Requests (BRs) with a Poisson arrival model having a fixed rate of $\lambda=30$ arrivals/hour. At an arrival of a BR at time $t$, the new bandwidth requirement of the LSP is calculated from $T_{\text {actual }}(i, j, t)$ according to (1).

\subsection{LSP Rerouting}

The online TE schemes investigated in this work are based on rerouting the LSPs to optimize the network performance. For each LSP to be constructed between a source-destination pair, a number of shortest paths are calculated beforehand using a $K$-shortest path algorithm. When a BR arrives for an LSP, among the paths belonging to that LSP, the best one is chosen according to the employed TE scheme and the LSP is (re)routed on that path. If there is not sufficient residual capacity along the path to accommodate the LSP, all of the available capacity is dedicated to the LSP and the amount of traffic that cannot be routed is assumed to be blocked. 
DREAM. DREAM is an alternate routing scheme which chooses the best path according the number of hop lengths and instantaneous residual capacities of the candidate paths. Each of the candidate paths is assigned a dynamic cost that is calculated by a cost function that utilizes the instantaneous residual capacity information. Then, the path with the minimum cost is chosen for rerouting the LSP. The cost function is designed in a way to choose the shorter paths when the network is lightly loaded and the paths with higher residual capacity when the network is heavily loaded. The cost function for path $p$, is given by:

$$
F_{\text {cost }}(p)=L_{p}+A^{u-\frac{C_{\text {residual }}^{p}}{C}}
$$

where, $L_{p}$ is the number of hops on path $p$ and $C_{\text {residual }}^{p}$ is the residual capacity of the path, i.e. the minimum residual capacity of the links (lightpaths) along the path, after the LSP is routed along that path and $C$ is the lightpath capacity. The performance of DREAM is tested on several networks with 10 nodes for various values of the parameters $A$ between 1 and 20 and $u$ between 0 and 1 , and the best results were obtained for the values of $A=10, u=0.5$.

The performance of DREAM is compared with various other schemes in the literature: Shortest Path routing (SP), Available Shortest Path routing (ASP) and Widest Shortest Path routing (WSP) [10,11]. In SP, no traffic engineering is applied and the LSPs are routed along the fixed shortest paths. ASP only consider the paths with sufficient residual capacity to accommodate the LSP. The shortest among these paths is chosen. If there are multiple such paths, one is chosen randomly. In WSP, similar to ASP, the shortest one is chosen among the paths with sufficient residual capacity. If there are multiple such paths, the one with the highest residual capacity is chosen. In both ASP and WSP, if there is a path with sufficient residual capacity to accommodate the LSP, it is rerouted along the path with the highest residual capacity and the amount of traffic that cannot be routed is assumed to be blocked.

Simulations are run for two different networks each having 10 nodes. The logical topologies are designed using the TS-MVTD algorithm with lightpath capacity $C=2 \operatorname{tgen}^{2}$ with 14 lightpaths and $C=4 \operatorname{tgen}^{2}$ with 23 lightpaths, respectively. These lightpath numbers are the minimum values needed by the TSMVTD algorithm to fulfill all the connection requests with the given capacity. The link capacities are then overprovisioned by $O P>0$, i.e. the capacities $C$ used in TS-VTDM are multiplied by $(1+O P)$, so that the uncertainty in the bandwidth requests can be accommodated.

The performances of the TE schemes are compared in terms of blocking ratio which is the ratio of the amount of blocked traffic to the total amount of offered traffic. The comparisons are made for $O P=10 \%$ and $25 \%$. The results are presented in Figs. 3 and 4 with networks of 14 and 23 lightpaths, respectively. In the figures, the $x$ axis is the value of the parameter $k$, which is the ratio of the standard deviation of the offered traffic to its expected value as explained in Section 2.1. The simulations are run for different values of $k$.

The significant difference in blocking ratios of the dynamic rerouting schemes and shortest path routing emphasizes the benefits of traffic engineering. Among 

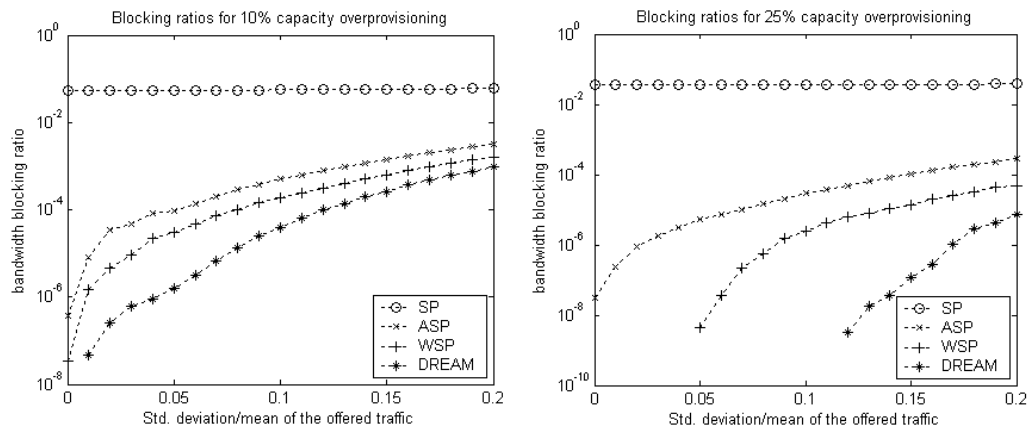

Fig. 3. Blocking ratios of the TE schemes for a network with 14 lightpaths
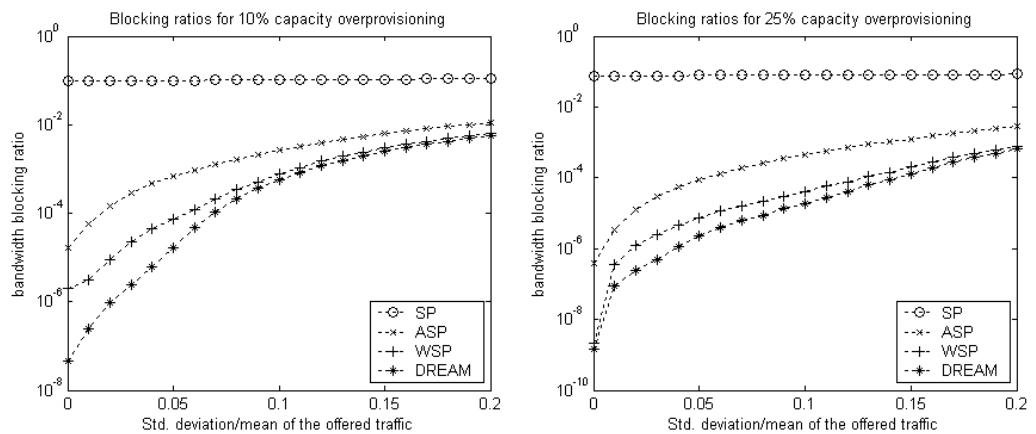

Fig. 4. Blocking ratios of the TE schemes for a network with 23 lightpaths

the dynamic rerouting schemes, ASP uses only the availability information of the paths (having or not having the sufficient residual capacity), while WSP and DREAM uses the full capacity information of the lightpaths. As a result, these two schemes outperform ASP. Among all the rerouting schemes, DREAM performs best.

\subsection{LSP Rerouting with Time Limit}

Although DREAM achieves the best blocking performance, it may result in a high frequency of LSP reroutings. Each time a BR arrives, the best route is calculated and the LSP is rerouted if the calculated route is different than the current one. To prevent excessive number of LSP reroutings, we introduce a minimum time limit between two consecutive reroutings of the same LSP. When a BR comes for an LSP, if sufficient time has not passed after the last rerouting of that LSP, only the bandwidth dedicated to that LSP is updated, however the route is not changed. We investigate the performance of DREAM with different time limits of 0.1 to 0.5 hour using the 23 lightpath network. Both the blocking 
ratios and the maximum number of reroutings per hour per LSP are depicted in Fig.5.
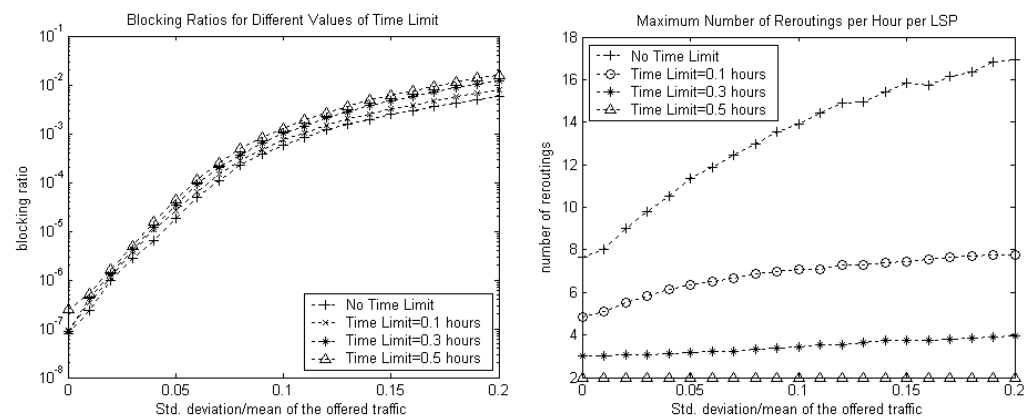

Fig. 5. Blocking ratios and number of reroutings for different values of time limit

As it can be seen from the results, DREAM can generate more than 16 reroutings per hour for an LSP in the worst case. Decreasing the number of reroutings by implementing a time limit causes an increase in the blocking ratio. However, it is possible to optimize the blocking ratio and the frequency of reroutings by choosing a time limit that keeps the frequency of reroutings in an acceptable range while not decreasing the throughput below a desired level.

\section{Online Traffic Engineering with Multiple Parallel LSPs}

If the total traffic between two nodes can be treated as the sum of multiple uninterruptible traffic flows, multiple parallel LSPs can be set up between the source and the sink nodes, and the total traffic can be distributed among these LSPs without splitting the individual flows over multiple LSPs. To model the traffic for the case of $L$ LSPs per node pair, the instantaneous traffic rate of each LSP is calculated independently by using the traffic generation function described in Section 2.1, with an expected value of $1 / L$ times the total expected value and a standard deviation of $1 / \sqrt{L}$ times the total standard deviation. Hence, the mean and the standard deviation of the total traffic for each node pair is same as the single LSP case. The arrivals of the BRs for the LSPs are modeled as independent Poisson processes with a rate of $\lambda / L$, where $\lambda$ is the arrival rate that is used in the case of single LSP per source-destination pair. The blocking ratios of the DREAM are presented for different values of $L$ in Fig. 6, for a network with 17 lightpaths and $O P=10 \%$.

The results show that increasing the number of the parallel LSPs brings a significant improvement on the network performance. Increasing the number of LSPs decreases flow granularity and DREAM can make better use of the residual capacities on the lightpaths at the expense of the additional control plane complexity. 


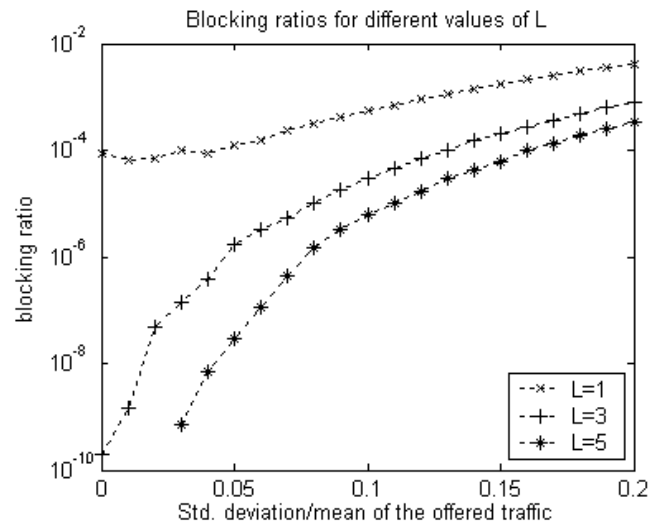

Fig. 6. Blocking ratios for different values of $L$

\subsection{Selecting the Overprovisioning Ratio}

In this part, we investigate how much overprovisioning is needed to guarantee a target blocking ratio for different number of parallel LSPs per node pair. Fig. 7 gives the required $O P$ as a function of the desired blocking probability for various values of $L$. These results are for a network having 10 nodes and 16 lightpaths. The lightpath capacities without overprovisioning are 3 tgen $^{2}$ and the traffic parameter $k$ is 0.15 . It is seen from the results that to keep the blocking ratio below $10^{-6}, O P>40 \%$ is required if a single LSP is set up between every node pair. If 5 parallel LSPs are set up, the required $O P$ decreases below $20 \%$. Using this figure, the network provider can choose the overprovisioning ratio according to the target blocking ratio and the number of parallel LSPs.

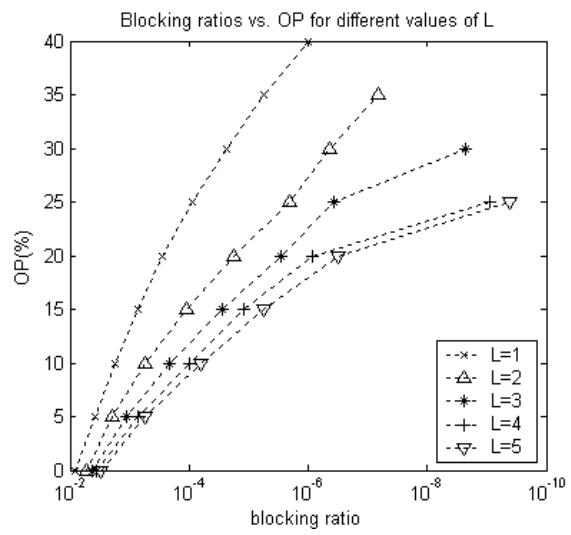

Fig. 7. Blocking ratio vs. $O P$ 


\section{Conclusions}

In this paper, we have presented a new and efficient TE scheme, DREAM, based on rerouting of the LSP connections. DREAM makes use of the available bandwidth information of the lightpaths. It favors the shorter paths when the network is lightly loaded and the paths with more residual capacity are preferred when the load on the lightpaths is higher.

The problem of designing a virtual topology for hourly changing traffic demands is also investigated and a heuristic is developed producing quite satisfactory solutions. On a topology produced by the proposed heuristic, DREAM provides a good blocking performance without topology updates and is superior to similar TE schemes also using the available bandwidth information.

Acknowledgements. This work is supported in part by the Scientific and Technological Research Council of Turkey (TUBITAK) under project EEEAG104E047 and by the IST-FP6 NoE project e-Photon/ONe+. Namik Sengezer is supported in part by the graduate scholarship of TUBITAK.

\section{References}

1. Xiao, X., Hannan, A., Bailey, B., Ni, L.: Traffic Engineering with MPLS in the Internet. Network, IEEE 14(2) (2000) 28-33

2. Awduche, D., Malcolm, J., Agogbua, J., O'Dell, M., McManus, J.: RFC2702: Requirements for Traffic Engineering Over MPLS. Internet RFCs (1999)

3. Awduche, D., Berger, L., Gan, D., Li, T., Srinivasan, V., Swallow, G.: RFC3209: RSVP-TE: Extensions to RSVP for LSP Tunnels. Internet RFCs (2001)

4. Bouillet, E., Labourdette, J., Ramamurthy, R., Chaudhuri, S.: Lightpath Reoptimization in Mesh Optical Networks. IEEE/ACM Transactions on Networking (TON) 13(2) (2005) 437-447

5. Bhatia, R., Kodialam, M.S., Lakshman, T.V.: Fast Network Re-optimization Schemes for MPLS and Optical networks. Computer Networks 50(3) (2006) 317331

6. Iovanna, P., Sabella, R., Settembre, M.: A Traffic Engineering System for Multilayer Networks Based on the GMPLS Paradigm. Network, IEEE 17(2) (2003) $28-37$

7. Pióro, M., Medhi, D.: Routing, Flow, and Capacity Design in Communication and Computer Networks. Elsevier/Morgan Kaufmann Amsterdam (2004)

8. Milbrandt, J., Menth, M., Kopf, S.: Adaptive Bandwidth Allocation: Impact of Traffic Demand Models for Wide Area Networks. In: 19th International Teletraffic Congress (ITC19), Beijing, China (2005)

9. Glover, F., Laguna, M.: Tabu Search. KLUWER ACADEMIC PUBL (1997)

10. Suri, S., Waldvogel, M., Warkhede, P.: Profile-Based Routing: A New Framework for MPLS Traffic Engineering. Quality of Future Internet Services, Lecture Notes in Computer Science 2156 (2001)

11. Wang, B., Su, X., Chen, C.: A New Bandwidth Guaranteed Routing Algorithm for MPLS Traffic Engineering. Proceedings of ICC 2 (2002) 1001-1005 